\title{
A simple multi-stable chaotic jerk system with two saddle-foci equilibrium points: Analysis, synchronization via backstepping technique and MultiSim circuit design
}

\author{
Aceng Sambas ${ }^{1}$, Sundarapandian Vaidyanathan ${ }^{2}$, Irene M. Moroz ${ }^{3}$, Babatunde Idowu ${ }^{4}$, \\ Mohamad Afendee Mohamed ${ }^{5}$, Mustafa Mamat ${ }^{6}$, W. S. Mada Sanjaya ${ }^{7}$ \\ ${ }^{1}$ Department of Mechanical Engineering, Universitas Muhammadiyah Tasikmalaya, Indonesia \\ ${ }^{2}$ Research and Development Centre, Vel Tech University, Chennai, Tamil Nadu, India \\ ${ }^{3}$ Mathematical Institute, University of Oxford, Andrew Wiles Building, ROQ, Oxford, United Kingdom \\ ${ }^{4}$ Department of Physics, Lagos State University, Ojo, Nigeria \\ ${ }^{5,6}$ Faculty of Informatics and Computing, Universiti Sultan Zainal Abidin, KualaTerengganu, Malaysia \\ ${ }^{7}$ Department of Physics, Universitas Islam Negeri Sunan Gunung Djati Bandung, Indonesia
}

\begin{tabular}{|c|c|}
\hline Article Info & ABSTRACT \\
\hline Article history: & This paper announces a new three-dimensional chaotic jerk system with two \\
\hline Received Jun 21, 2020 & $\begin{array}{l}\text { saddle-focus equilibrium points and gives a dynamic analysis of the } \\
\text { properties of the jerk system such as Lyapunov exponents, phase portraits, }\end{array}$ \\
\hline Revised Dec 13, 2020 & Kaplan-Yorke dimension and equilibrium points. By modifying the Genesio- \\
\hline Accepted Jan 8, 2021 & $\begin{array}{l}\text { Tesi jerk dynamics (1992), a new jerk system is derived in this research } \\
\text { study. The new jerk model is equipped with multistability and dissipative }\end{array}$ \\
\hline Keywords: & $\begin{array}{l}\text { chaos with two saddle-foci equilibrium points. By invoking backstepping } \\
\text { technique, new results for synchronizing chaos between the proposed jerk }\end{array}$ \\
\hline $\begin{array}{l}\text { Backstepping control } \\
\text { Chaotic system } \\
\text { Electronic circuit }\end{array}$ & $\begin{array}{l}\text { models are successfully yielded. MultiSim software is used to implement a } \\
\text { circuit model for the new jerk dynamics. A good qualitative agreement has } \\
\text { been shown between the MATLAB simulations of the theoretical chaotic } \\
\text { jerk model and the MultiSIM results. }\end{array}$ \\
\hline
\end{tabular}

Jerk system

Synchronization
This is an open access article under the CC BY-SA license.

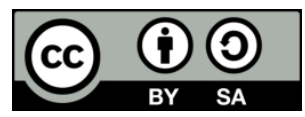

\section{Corresponding Author:}

Mohamad Afendee Mohamed

Faculty of Informatics and Computing

Universiti Sultan Zainal Abidin

Kampung Gong Badak, 21300, Terengganu, Malaysia

Email: mafendee@unisza.edu.my

\section{INTRODUCTION}

Chaotic systems are applicable in a wide range of research classifications such as jerk systems [1,2], robotics [3, 4], neuron models [5, 6], oscillators [7, 8], circuits [9-11], biological systems [12, 13], chemical systems $[14,15]$, and memristors $[16,17]$. In view of their attractive triangular structure, considerable numbers of papers have been published on the chaos jerk models [18-20]. A general form of the autonomous jerk models can be exhibited by a system model as (1).

$$
\left\{\begin{array}{l}
\dot{\xi}_{1}=\xi_{2} \\
\dot{\xi}_{2}=\xi_{3} \\
\dot{\xi}_{3}=f\left(\xi_{1}, \xi_{2}, \xi_{3}\right)
\end{array}\right.
$$


By modifying the Genesio-Tesi jerk dynamics (1992) with the introduction of a quadratic nonlinear term, a new jerk system is derived in this research study. The modelling is detailed in section 2 . The new jerk model is equipped with multistability and dissipative chaos with two saddle-foci equilibrium points as described in section 3. By invoking backstepping technique, new results for synchronizing chaos between the proposed jerk models are successfully yielded. Section 3 comprises the new backstepping based control results. In section 4, MultiSim software is used to implement a circuit model for the new dynamics.

\section{A NEW CHAOTIC JERK SYSTEM}

In 1992, Genesio and Tesi [20] proposed the mechanical model.

$$
\left\{\begin{array}{l}
\dot{\xi}_{1}=\xi_{2} \\
\dot{\xi}_{2}=\xi_{3} \\
\dot{\xi}_{3}=-a \xi_{1}-b \xi_{2}-c \xi_{3}+\xi_{1}^{2}
\end{array}\right.
$$

In the dynamical model (2), $\xi=\left(\xi_{1}, \xi_{2}, \xi_{3}\right)$ stands for the three-dimensional phase vector, while $(a, b, c)$ represents the vector of system parameters. Genesio and Tesi [20] established by applying Lyapunov exponent analysis [21] for the jerk model (2). The model (2) has chaos nature if we take $(a, b, c)=(1.0,1.1,0.44)$ and the initial phase vector $\xi(0)=(0.3,0.1,0.2)$ as the Lyapunov exponents spectrum can be calculated using [21] as (3).

$$
\rho_{1}=0.1052, \rho_{2}=0, \rho_{3}=-0.5452
$$

The presence of $\rho_{1}>0$ in the LE spectrum (3) establishes the chaos nature of the jerk model (2). By adding a quadratic nonlinearity to the Genesio-Tesi chaotic model (2), a new three-dimensional dynamical model is derived as (4).

$$
\left\{\begin{array}{l}
\dot{\xi}_{1}=\xi_{2} \\
\dot{\xi}_{2}=\xi_{3} \\
\dot{\xi}_{3}=-a \xi_{1}-b \xi_{2}-c \xi_{3}+d \xi_{1} \xi_{2}+\xi_{1}^{2}
\end{array}\right.
$$

In the dynamical model (4), $\xi=\left(\xi_{1}, \xi_{2}, \xi_{3}\right)$ stands for the three-dimensional phase vector, while $(a, b, c, d)$ represents the vector of system parameters. The new dynamical model (4) has chaos nature if we take $(a, b, c, d)=(1.3,1.3,0.5,0.1)$ and the initial phase vector $\xi(0)=(0.3,0.1,0.2)$ as the Lyapunov exponents spectrum can be calculated using [21] as (5).

$$
\rho_{1}=0.1080, \rho_{2}=0, \rho_{3}=-0.6080
$$

The presence of $\rho_{1}>0$ in the LE spectrum (5) establishes the chaos nature of the jerk model (2). A comparison of the Genesio-Tesi jerk model (2) and the modified jerk model (4) can be encapsulated as follows. The Genesio-Tesi jerk model (2) has five linear terms and a single quadratic nonlinearity, while the modified model (4) has five linear terms and 2 quadratic nonlinearities. The maximal values of the Lyapunov exponents of the models (2) and (4) are easily seen as $p_{1}=0.1052$ and $p_{1}=0.1080$ respectively. Furthermore, the model (4) has complex behavior such as multi-stability with coexistence of chaos attractors. For finding the balance points of the model (4), we seek the roots of the system given as (6a), (6b), (6c):

$$
\xi_{2}=0
$$




$$
\xi_{3}=0
$$

$$
-a \xi_{1}-b \xi_{2}-c \xi_{3}+d \xi_{1} \xi_{2}+\xi_{1}^{2}=0
$$

By finding the roots of the system (6), we arrive at two balance points of the model (4) as (7).

$$
\mathrm{T}_{0}=(0,0,0) \text { and } \mathrm{T}_{1}=(a, 0,0)
$$

In the chaos situation, $(a, b, c, d)=(1.3,1.3,0.5,0.1)$. Thus, the balance points for the jerk model (4) are derived as $\mathrm{T}_{0}=(0,0,0)$ and $\mathrm{T}_{1}=(1.3,0,0)$. Calculating the spectral values of the linearization matrix of the jerk model (4) at $\mathrm{T}_{0}$, we get:

$$
\mu_{1}=-0.8275, \mu_{2,3}=0.1637 \pm 1.2427
$$

Calculating the spectral values of the linearization matrix of the jerk model (4) at $T_{1}$, we get:

$$
\mu_{1}=0.6671, \mu_{2,3}=-0.5836 \pm 1.2681 i
$$

This shows that $T_{0}=(0,0,0)$ and $T_{1}=(1.3,0,0)$ are saddle-foci, unstable balance points of the modified model (4). Figure 1 represents various signal plots of the jerk model (4) in the 2-D planes.

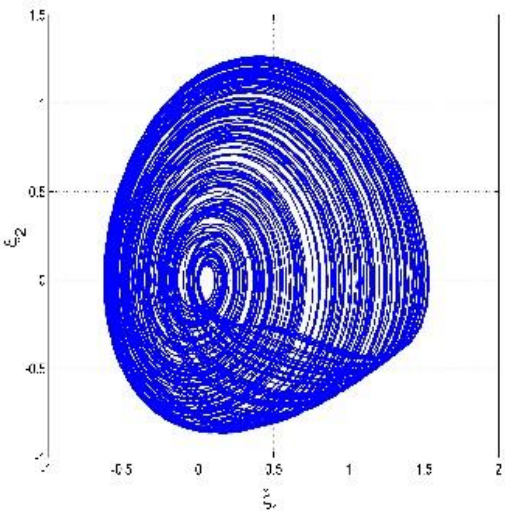

(a)

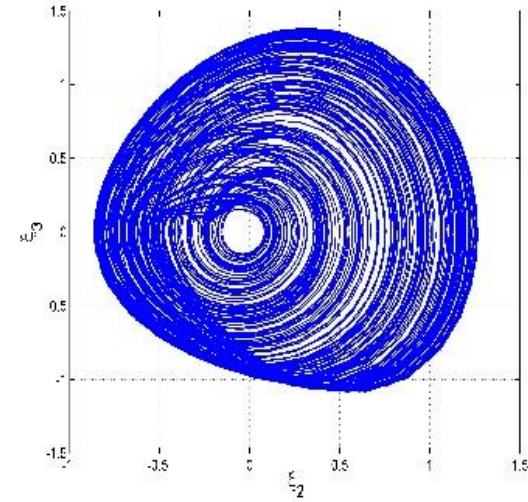

(b)

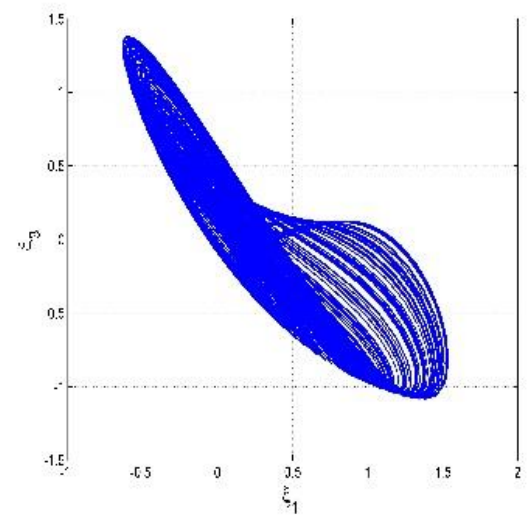

(c)

Figure 1. Two-dimensional signal plots of the jerk model (4) in the coordinate planes 
It is worthwhile to observe that the jerk model (4) exhibits multistability phenomenon, which is the coexistence of chaos attractors when choosing different initial phase vectors $[22,23]$. The parameter vector is fixed as in the chaos situation, i.e. $(a, b, c, d)=(1.3,1.3,0.5,0.1)$. Two initial phase vectors are selected as $\xi_{0}=(0.3,0.1,0.2)$ and $\eta_{0}=(-0.5,0,-0.5)$, and the corresponding signal plots of the jerk model (4) are depicted in blue and red colors, respectively. Figure 2 illustrates the multistability of the jerk model (4).

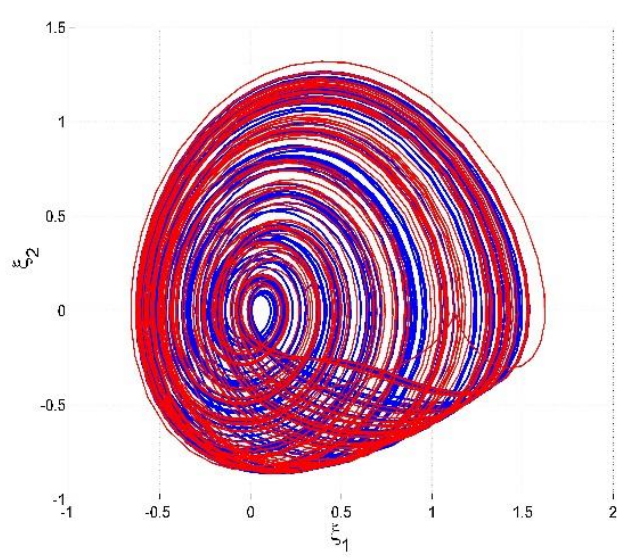

(a)

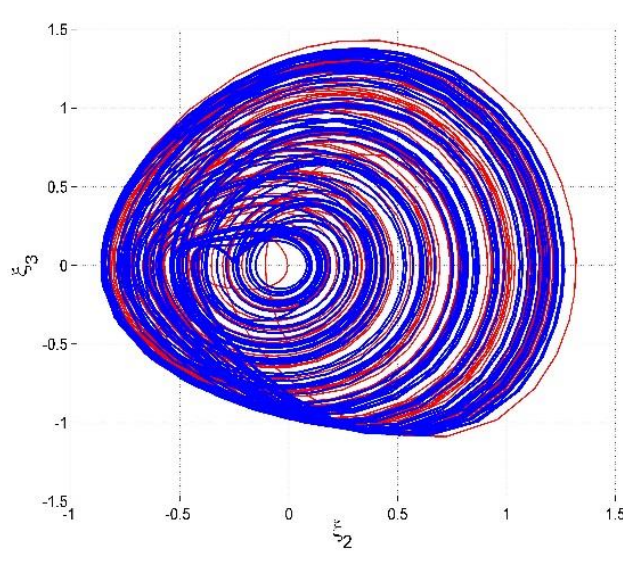

(b)

Figure 2. Multistability of the jerk model (4) with $(a, b, c, d)=(1.3,1.3,0.5,0.1)$ and initial phase vectors $\xi_{0}=(0.3,0.1,0.2)$ (blue color orbit) and $\eta_{0}=(-0.5,0,-0.5)$ (red color orbit)

\section{BIFURCATION ANALYSIS OF THE NEW JERK SYSTEM}

For the modified jerk model (4), we consider general positive values for $a, b, c$, $d$. The linear stability of each balance point in (9) is determined from the eigenvalues of the associated characteristic equation. For the trivial balance point $\mathrm{T}_{0}$, we have

$$
\lambda^{3}+c \lambda^{2}+b \lambda+a=0
$$

A steady state bifurcation $(\lambda=0)$ can occur only when $a=0$. For a Hopf bifurcation, we set $\lambda=i \omega$ in (10), which yields:

$$
(i \omega)^{3}+c(i \omega)^{2}+b(i w)+a=0
$$

Simplififying (11) and rearranging terms, it is deduced that:

$$
\left(a-c \omega^{2}\right)+i \omega\left(b-\omega^{2}\right)=0 .
$$

By equating the imaginary and real parts of both sides of (12), it is seen that:

$$
\omega_{R}^{2}=\frac{a}{c}>0, \quad \omega_{I}^{2}=b>0
$$

such that $\frac{a}{c}=b$.

For the nontrivial balance point $T_{1}$, where $\xi_{1}=a$, the characteristic equation becomes: 


$$
\Lambda^{3}+c \Lambda^{2}+(b-d a) \Lambda-a=0
$$

A steady state bifurcation occurs when $a=0$. Thus, we have an exchange of stabilities beween $\mathrm{T}_{0}$ and $\mathrm{T}_{1}$. However, for a Hopf bifurcation, we set $\Lambda=i \Omega$ in (14), which yields:

$$
(i \Omega)^{3}+c(i \Omega)^{2}+(b-d a)(i \Omega)-a=0 .
$$

Simplifying (15) and rearranging terms, it is deduced that:

$$
-\left(a+c \Omega^{2}\right)+i \Omega\left(b-d a-\Omega^{2}\right)=0 .
$$

By equating the imaginary and real parts of both sides of (12), we deduce as (17):

$$
\Omega_{R}^{2}=-\frac{a}{c}>0, \Omega_{I}^{2}=b-d a>0
$$

provided $b=d a-\frac{a}{c}$.

Since all four parameters are required to be positive, the first condition in (17) cannot be met. Thus, a Hopf bifurcation for $T_{1}$ is not possible. We can verify these results by constructing bifurcation transition diagrams. We choose $a$ as the bifurcation parameter, fixing the remaining parameters at their prescribed values. The blue dots show the bifurcation plot as $a$ decreases from $a=1.3$ using $\xi_{0=}\left(\begin{array}{lll}0.1 & 0.1 & 0.1\end{array}\right)$ as the starting value. There is a period-doubling bifurcation to a period-2 cycle at $a \approx 1.11$ and another perioddoubling bifurcation to a period-4 cycle at $a \approx 1.186$. The periodic solution loses stability to a Hopf bifurcation at $a=6.5$, which is precisely the Hopf bifurcation condition of $a=b c$, when $b=1.3$ and $c=0.5$. When we varied $a$ beyond $a=1.3$, we were able to increase $a$ up to $a \approx 1.338$, before the system variables became unbounded. Figure 3 displays the bifurcation diagram plot of the maximum values of $\xi_{1}$ over each cycle as $a$ varies between $a=0.6$ and $a=1.338$ for the initial condition $\xi_{0=}\left(\begin{array}{lll}0.1 & 0.10 .1\end{array}\right)$.

Figure 4 demonstrates a comparison of the bifurcation transition plots as $a$ is decreased, starting from two different starting values $\xi_{0}=\left(\begin{array}{lll}0.1 & 0.1 & 0.1\end{array}\right)$ (blue dots) and $\xi_{0}=\left(\begin{array}{lll}-0.5 & 0 & -0.5\end{array}\right)$ (red dots). There are periodic windows within the chaotic regimes in each case, but some are only visible with one of the initial conditions, such as the periodic window in $1.31 \leq a \leq 1.314$ for the first set of initial conditions.

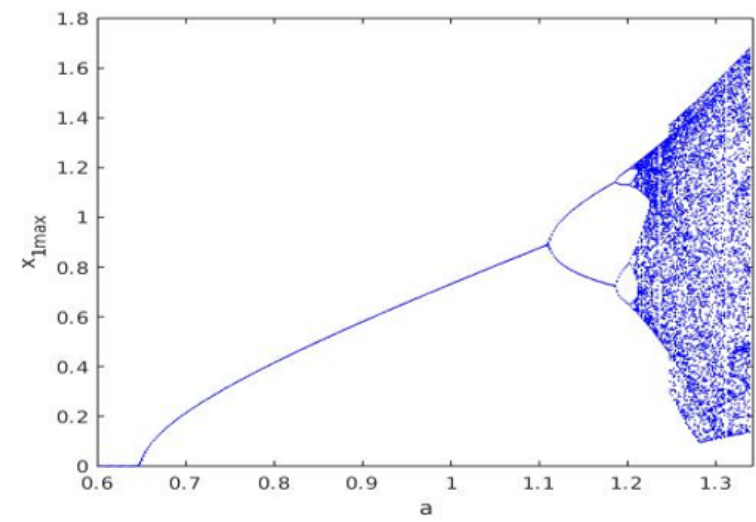

Figure 3. Bifurcation transition simulation diagram of the maximum values of $\xi_{1}$ over each cycle as $a$ varies between $a=0.6$ and $a=1.338$ for $\xi_{0=}\left(\begin{array}{lll}0.1 & 0.1 & 0.1\end{array}\right)$. Note $\left(X_{1 \max }=\xi_{1 \max }\right)$

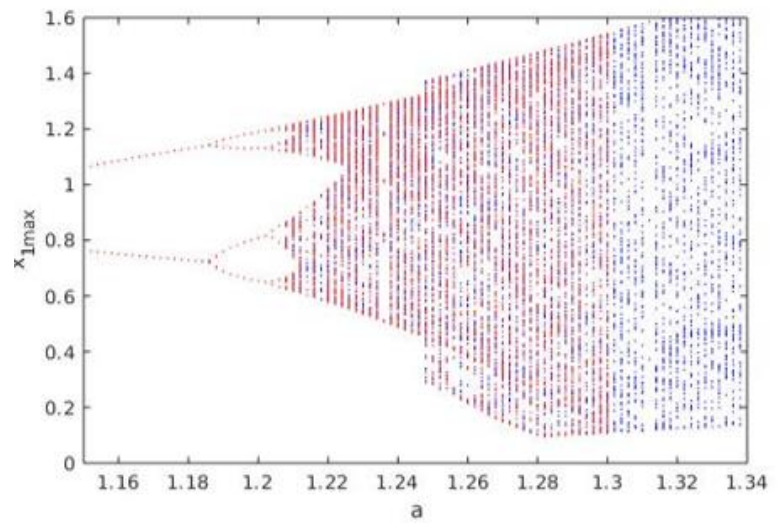

Figure 4. Bifurcation transition simulation diagram of the maximum values of $\xi_{1}$ over each cycle as $a$

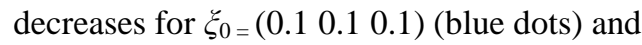
$\xi_{0=}(-0.50-0.5)$ (red dots). Note $\left(X_{1 \max }=\xi_{1 \max }\right)$ 


\section{GLOBAL CHAOS SYNCHRONIZATION OF THE NEW JERK SYSTEMS VIA ACTIVE \\ BACKSTEPPING CONTROL}

With the use of backstepping technique, new results are encapsulated in this section for the synchronising design of the new chaos models envisioned as leader and follower systems. The leader system is specified by the new chaos model dynamics given in (18).

$$
\left\{\begin{array}{l}
\dot{\alpha}_{1}=\alpha_{2} \\
\dot{\alpha}_{2}=\alpha_{3} \\
\dot{\alpha}_{3}=-a \alpha_{1}-b \alpha_{2}-c \alpha_{3}+d \alpha_{1} \alpha_{2}+\alpha_{1}^{2}
\end{array}\right.
$$

Furthermore, the follower system is specified by the controlled chaos model dynamics given in (19).

$$
\left\{\begin{array}{l}
\dot{\beta}_{1}=\beta_{2} \\
\dot{\beta}_{2}=\beta_{3} \\
\dot{\beta}_{3}=-a \beta_{1}-b \beta_{2}-c \beta_{3}+d \beta_{1} \beta_{2}+\beta_{1}^{2}+u
\end{array}\right.
$$

In the systems (18) and (19), $\alpha=\left(\alpha_{1}, \alpha_{2}, \alpha_{3}\right)$ and $\beta=\left(\beta_{1}, \beta_{2}, \beta_{3}\right)$ are the states. Also, $u$ is a backstepping controller that is to be determined in this section. The action of $u$ is to enable synchronization of the respective phases of the jerk models (18) and (19). For this purpose, we shall define synchronizing error between the respective phases of the jerk models (18) and (19) as being (20).

$$
\left\{\begin{array}{l}
\varepsilon_{1}=\beta_{1}-\alpha_{1} \\
\varepsilon_{2}=\beta_{2}-\alpha_{2} \\
\varepsilon_{3}=\beta_{1}-\alpha_{3}
\end{array}\right.
$$

The error phases satisfy the system of differential equations as given in (21).

$$
\left\{\begin{array}{l}
\dot{\varepsilon}_{1}=\varepsilon_{2} \\
\dot{\varepsilon}_{2}=\varepsilon_{3} \\
\dot{\varepsilon}_{3}=-a \varepsilon_{1}-b \varepsilon_{2}-c \varepsilon_{3}+d\left(\beta_{1} \beta_{2}-\alpha_{1} \alpha_{2}\right)+\left(\beta_{1}^{2}-\alpha_{1}^{2}\right)+u
\end{array}\right.
$$

Next, we shall outline a main backstepping control result providing a compact formula for the feedback control $u$ which will achieve the desired synchronization between the leader and follower models (18) and (19).

Theorem 1. The backstepping feedback control law mentioned by

$$
u=-(3-a) \varepsilon_{1}-(5-b) \varepsilon_{2}-(3-c) \varepsilon_{3}-d\left(\beta_{1} \beta_{2}-\alpha_{1} \alpha_{2}\right)-\beta_{1}^{2}+\alpha_{1}^{2}-L \mu_{3}
$$

where $L>0$ is a controller gain and $\mu_{3}=2 \varepsilon_{1}+2 \varepsilon_{2}+\varepsilon_{3}$, renders global asymptotic synchronization between the states of the leader and follower chaos models (18) and (19).

Proof. The assertion of Theorem 1 shall be established with an application of Lyapunov stability theory [24-27]. We start the proof by defining a scalar Lyapunov function as (23):

$$
W_{1}\left(\mu_{1}\right)=0.5 \mu_{1}^{2}
$$

where $\mu_{1}=\varepsilon_{1}$.

The time-derivative of $W_{1}$ along the error dynamics (21) can be easily found as being (24): 
$\dot{V}_{1}=\xi_{1} \dot{\xi}_{1}=e_{1} e_{2}=-\xi_{1}^{2}+\xi_{1}\left(e_{1}+e_{2}\right)$

Next, we define

$$
\mu_{2}=\varepsilon_{1}+\varepsilon_{2}
$$

We can express (24) using (25) as (26):

$$
\dot{W}_{1}=-\mu_{1}^{2}+\mu_{1} \mu_{2}
$$

Next, we propose the Lyapunov function

$$
W_{2}\left(\mu_{1}, \mu_{2}\right)=W_{1}\left(\mu_{1}\right)+0.5 \xi_{2}^{2}=0.5\left(\mu_{1}^{2}+\mu_{2}^{2}\right)
$$

The time-derivative of $W_{2}$ along the error dynamics (21) can be found as (28):

$$
\dot{W}_{2}=-\mu_{1}^{2}-\mu_{2}^{2}+\mu_{2}\left(2 \varepsilon_{1}+2 \varepsilon_{2}+\varepsilon_{3}\right)
$$

Next, we define

$$
\mu_{3}=2 \varepsilon_{1}+2 \varepsilon_{2}+\varepsilon_{3}
$$

We can express (28) using (29) as (30):

$$
\dot{W}_{2}=-\mu_{1}^{2}-\mu_{2}^{2}+\mu_{2} \mu_{3}
$$

Finally, we propose the Lyapunov function

$$
W\left(\mu_{1}, \mu_{2}, \mu_{3}\right)=0.5\left(\mu_{1}^{2}+\mu_{2}^{2}+\mu_{3}^{2}\right)
$$

It is a straightforward calculation to verify that $W$ is a positive definite function on $R^{3}$. The timederivative of $W$ along the error dynamics (21) can be found as (32):

$$
\dot{W}=-\mu_{1}^{2}-\mu_{2}^{2}-\mu_{3}^{2}+\mu_{3}\left(\mu_{3}+\mu_{2}+\dot{\mu}_{3}\right)=-\mu_{1}^{2}-\mu_{2}^{2}-\mu_{3}^{2}+\mu_{3}(R)
$$

where

$$
R=\mu_{3}+\mu_{2}+\dot{\mu}_{3}=\mu_{3}+\mu_{2}+\left(2 \dot{\varepsilon}_{1}+2 \dot{\varepsilon}_{2}+\dot{\varepsilon}_{3}\right)
$$

A simple calculation yields the result (34):

$$
R=(3-a) \varepsilon_{1}+(5-b) \varepsilon_{2}+(3-c) \varepsilon_{3}+d\left(\beta_{1} \beta_{2}-\alpha_{1} \alpha_{2}\right)+\beta_{1}^{2}-\alpha_{1}^{2}+u
$$

When we substitute the active feedback law (22) into (34), we arrive at (35):

$$
R=-L \mu_{3}
$$

Substituting the value of $R$ from (35) into (32), we derive as (36):

$$
\dot{W}=-\mu_{1}^{2}-\mu_{2}^{2}-(1+L) \mu_{3}^{2}
$$


Since $\dot{W}$ is a quadratic and negative definite function on $R^{3}$, we conclude that the error variables $\varepsilon_{1}(t), \varepsilon_{2}(t)$ and $\varepsilon_{3}(t)$ tend to zero as $t \rightarrow \infty$ for all values of $\varepsilon_{i}(0) \in R$ for $i=1,2,3$.

For the computer simulations in MATLAB, the parameters of the jerk models (18) and (19) are set as in the chaotic case, viz. $(a, b, c, d)=(1.3,1.3,0.5,0.1)$. For fast convergence, we choose $L=20$.

The initial state of the leader model $(18)$ is picked as $\alpha(0)=(1.60 .4-1.3)$.

The initial state of the follower system (19) is picked as $\beta(0)=(2.8-0.71 .2)$.

Figure 5 pinpoints the complete synchronization of the new jerk models represented by (18) and (19). Furthermore, Figure 6 depicts the time-history of the synchronization error between the new chaos jerk models (18) and (19).
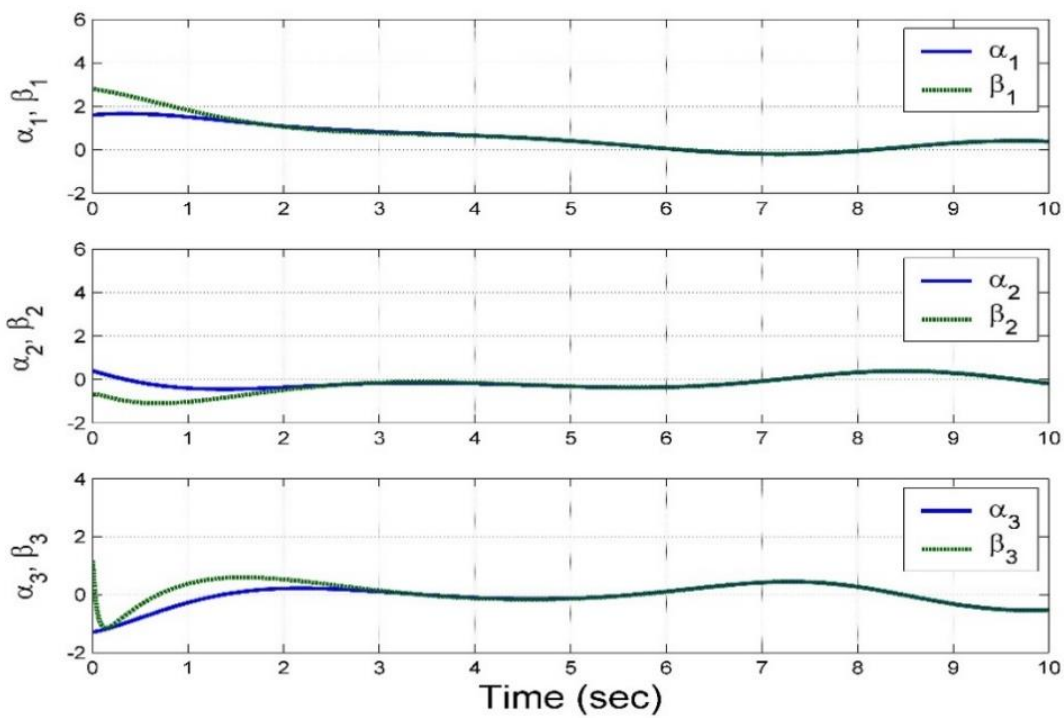

Figure 5. Backstepping-based asymptotic synchronization of the chaos models (18) and (19)

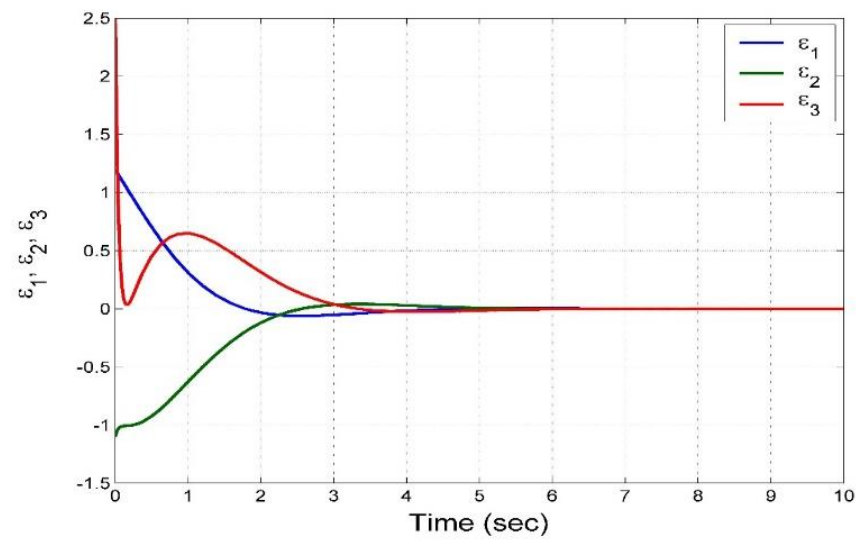

Figure 6. Time-plot of the backstepping-based synchronization error between the chaos models (18) and (19)

\section{MULTISIM CIRCUIT SIMULATION OF THE NEW CHAOTIC JERK MODEL}

A MultiSim circuit model of the new chaos jerk model (4) is implemented by using off-the-shelf components such as operational amplifiers, capacitors, resistors, and analog multipliers. The MultiSim circuit in Figure 7 has been designed by applying the general approach with operational amplifiers. Thus, the variables $\xi_{1}, \xi_{2}, \xi_{3}$ of the jerk model (4) are the voltages across the capacitor $C_{1}, C_{2}$ and $C_{3}$, respectively. Note: $\xi_{1}=X_{1}, \xi_{2}=X_{2}$ and $\xi_{3}=X_{3}$. By applying Kirchhoff's circuit laws, the corresponding circuital equations of the designed circuit can be expressed as given (37). 


$$
\left\{\begin{array}{l}
\dot{\xi}_{1}=\frac{1}{C_{1} R_{1}} \xi_{2} \\
\dot{\xi}_{2}=\frac{1}{C_{2} R_{2}} \xi_{3} \\
\dot{\xi}_{3}=-\frac{1}{C_{3} R_{3}} \xi_{1}-\frac{1}{C_{3} R_{4}} \xi_{2}-\frac{1}{C_{3} R_{5}} \xi_{3}+\frac{1}{10 C_{3} R_{6}} \xi_{1} \xi_{2}+\frac{1}{10 C_{3} R_{7}} \xi_{1}^{2}
\end{array}\right.
$$

The power supplies of all active devices are $\pm 15 V_{\mathrm{DC}}$ and the TL082CD operational amplifiers are used in this work. The values of components in Figure 8 are chosen to match the parameters of system (37) as: $R_{1}=R_{2}=R_{6}=R_{7}=R_{8}=R_{9}=R_{10}=R_{11}=R_{12}=R_{13}=100 \mathrm{k} \Omega, R_{3}=R_{4}=76.92 \quad \mathrm{k} \Omega, \quad R_{5}=200 \quad \mathrm{k} \Omega, R_{7}=10 \quad \mathrm{k} \Omega$ and $C_{1}=C_{2}=C_{3}=1 \mathrm{nF}$. The designed circuit has been examined by MultiSim software and results are described in Figure 7. It is easy to see a good agreement between the MultiSim simulation as shown in Figure 8 and MATLAB simulation as shown in Figure 1.

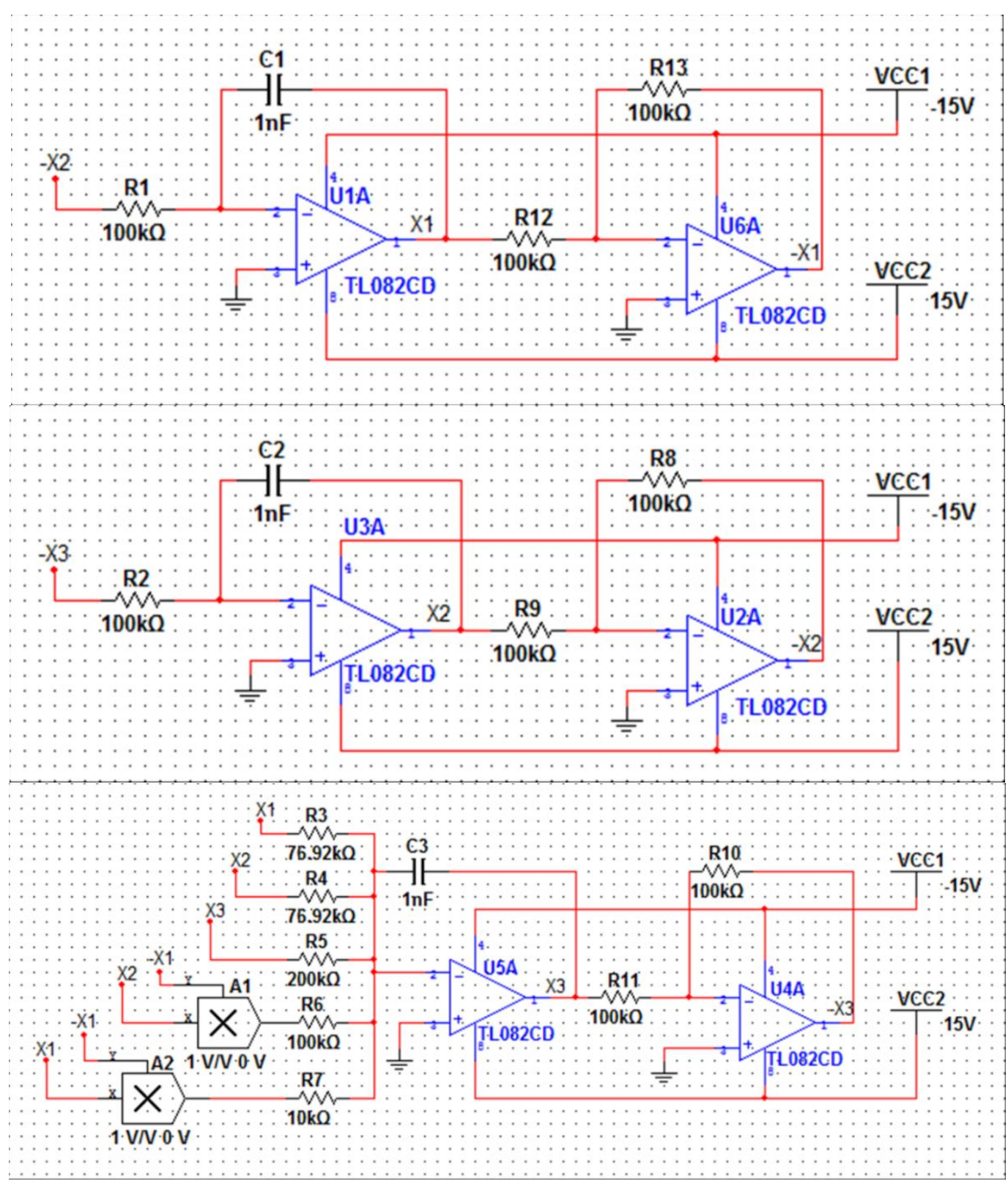

Figure 7. The schematic diagram of the MultiSim circuit of new jerk model (37) 


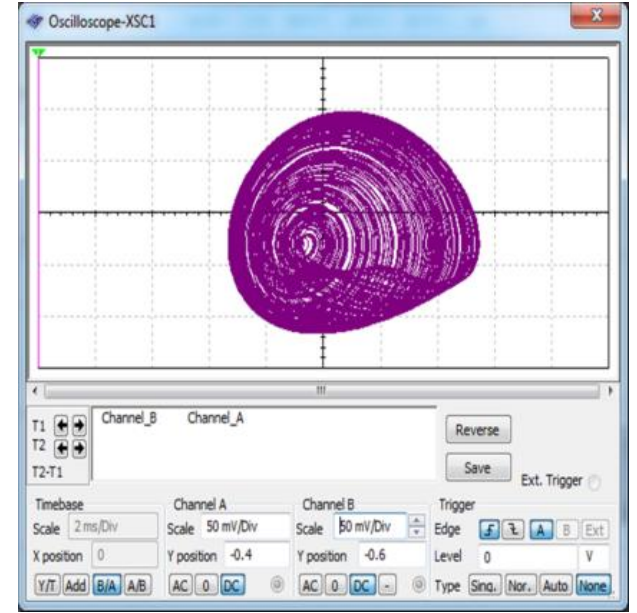

(a)

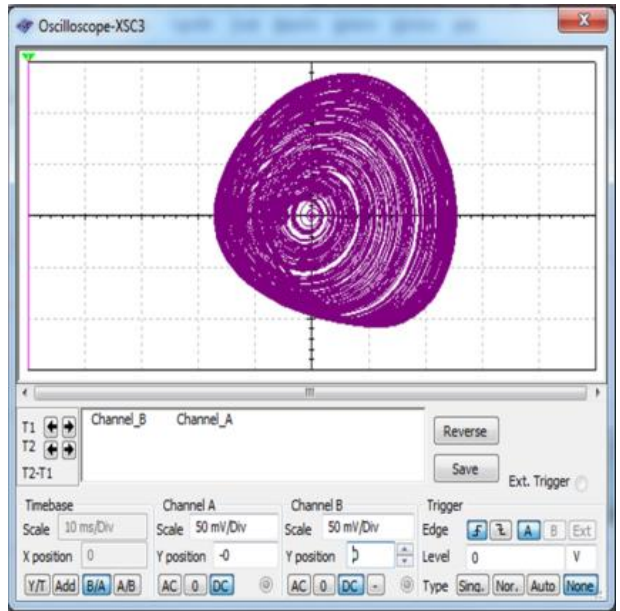

(b)

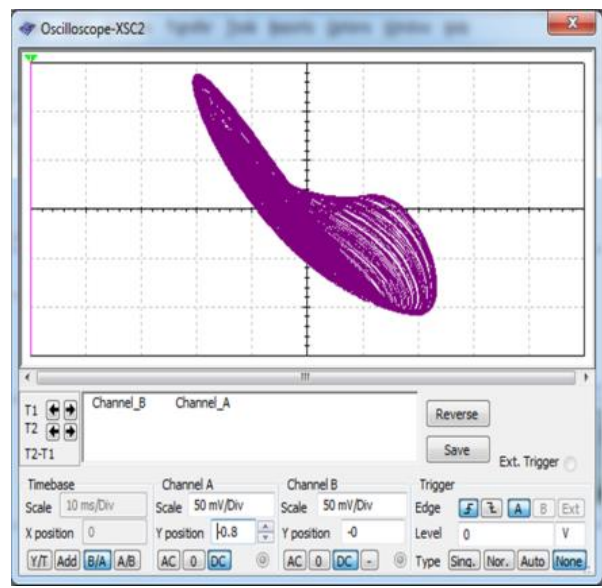

(c)

Figure 8. Phase potraits of the MultiSim simulation of the jerk model (37) in the (a) $\left(\xi_{1}, \xi_{2}\right)$-plane,

(b) $\left(\xi_{2}, \xi_{3}\right)$-plane and (c) $\left(\xi_{1}, \xi_{3}\right)$-plane

\section{CONCLUSION}

In this paper, by modifying the Genesio-Tesi jerk dynamics (1992), a new jerk system model was derived and its dynamical properties were analyzed in detail. It was shown that the new jerk model has with multistability and dissipative chaos with two saddle-foci balance points. By invoking backstepping technique, new results for synchronizing chaos between the proposed jerk models were successfully yielded and proved using Lyapunov stability theory. Furthermore, MultiSim software was used in order to implement a circuit model for the new jerk dynamics. The control application and circuit implementation of the new jerk model have useful applications in engineering such as secure communications, and crypto-devices.

\section{ACKNOWLEDGEMENTS}

This project is funded by the Center for Research Excellence, Incubation Management Center, Universiti Sultan Zainal Abidin.

\section{REFERENCES}

[1] Alligood, K. T., Sauer, T. D., and Yorke, J. A., “Chaos,” New York, Springer, 1996.

[2] J. C. Sprott, "Elegant chaos: algebraically simple chaotic flows," Singapore, World Scientific, 2010.

[3] Vaidyanathan, S., Sambas, A., Mamat, M., and Sanjaya, M., "A new three-dimensional chaotic system with a hidden attractor, circuit design and application in wireless mobile robot," Archives of Control Sciences, vol. 27, pp. 541-554, 2017. 
[4] Arboleda, E. R., Balaba, J. L., and Espineli, J. C. L., "Chaotic rivest-shamir-adlerman algorithm with data encryption standard scheduling," Bulletin of Electrical Engineering and Informatics, vol. 6, pp. 219-227, 2017.

[5] Vafaei, V., Kheiri, H., and Akbarfam, A. J., "Synchronization of fractional-order chaotic systems with disturbances via novel fractional integer integral sliding mode control and application to neuron models," Mathematical Methods in the Applied Sciences, vol. 42, no. 8, pp. 2761-2773, 2019.

[6] Cejnar, P., Vyšata, O., Kukal, J., Beránek, M., Vališ, M., and Procházka, A., "Simple capacitor-switch model of excitatory and inhibitory neuron with all parts biologically explained allows input fire pattern dependent chaotic oscillations," Scientific Reports, vol. 10, no. 1, pp. 1-18, 2020.

[7] S. Vaidyanathan, "Output regulation of the unified chaotic system," Communications in Computer and Information Science, vol. 198, pp. 1-9, 2011.

[8] Ren, G., Xue, Y., Li, Y., and Ma, J., "Field coupling benefits signal exchange between Colpitts systems," Applied Mathematics and Computation, vol. 342, pp. 45-54, 2019.

[9] A. Sambas et al., "A new double-wing chaotic system with coexisting attractors and line equilibrium: Bifurcation analysis and electronic circuit simulation," IEEE Access, vol. 7, pp. 115454-115462, 2019.

[10] A. Sambas et al., "A new chaotic system with line of equilibria: dynamics, passive control and circuit design," International Journal of Electrical and Computer Engineering, vol. 9, pp. 2365-2376, 2019.

[11] A. Sambas et al., "A novel chaotic system with two circles of equilibrium points: Multistability, electronic circuit and FPGA realization," Electronics, vol. 8, p. 1211, 2019.

[12] S. Vaidyanathan et al., "A new biological snap oscillator: its modelling, analysis, simulations and circuit design," International Journal of Simulation and Process Modelling, vol. 13, no. 5, pp. 419-432, 2018.

[13] V. Rai and R. K. Upadhyay, "Chaotic population dynamics and biology of the top-predator," Chaos, Solitons \& Fractals, vol. 21, no. 5, pp. 1195-1204, 2004.

[14] T. S. Lim, Y. Lu, and J. H. Nolen, "Quantitative propagation of chaos in a bimolecular chemical reaction-diffusion model,” SIAM Journal on Mathematical Analysis, vol. 52, no. 2, pp. 2098-2133, 2020.

[15] S. Vaidyanathan, "Synchronization of Tokamak systems with symmetric and magnetically confined plasma via adaptive control," International Journal of ChemTech Research, vol. 8, no. 6, pp. 818-827, 2015.

[16] Li, Y., Huang, X., Song, Y., and Lin, J., "A new fourth-order memristive chaotic system and its generation," International Journal of Bifurcation and Chaos, vol. 25, no. 11, 2015, Art. no. 1550151.

[17] Bao, B. C., Bao, H., Wang, N., Chen, M., and Xu, Q., "Hidden extreme multistability in memristive hyperchaotic system," Chaos, Solitons \& Fractals, vol. 94, pp. 102-111, 2017.

[18] Vaidyanathan, S., Sambas, A., Mamat, M., and W. S. Mada, "Analysis, synchronisation and circuit implementation of a novel jerk chaotic system and its application for voice encryption," International Journal of Modelling, Identification and Control, vol. 28, pp. 153-166, 2017.

[19] J. Kengne et al., "Nonlinear behavior of a novel chaotic jerk system: antimonotonicity, crises, and multiple coexisting attractors," International Journal of Dynamics and Control, vol. 6, no. 2, pp. 468-485, 2018.

[20] R. Genesio and A. Tesi, "Harmonic balance methods for the analysis of chaotic dynamics in nonlinear systems," Automatica, vol. 28, pp. 531-548, 1992.

[21] Wolf, A., Swift, J. B., Swinney, H. L., and Vastano, J. A., "Determining Lyapunov exponents from a time series," Physica D: Nonlinear Phenomena, vol. 16, pp. 285-317, 1985.

[22] Vaidyanathan, S., Sambas, A., Zhang, S., Zeng, Y., Mohamed, M. A., and Mamat, M., "A new two-scroll chaotic system with two nonlinearities: dynamical analysis and circuit simulation," Telkomnika Telecommunication, Computing, Electronics and Control, vol. 17, no. 5, pp. 2465-2474, 2019.

[23] A. Sambas et al., "Multistability in a Novel Chaotic System with Perpendicular Lines of Equilibrium: Analysis, Adaptive Synchronization and Circuit Design,” Engineering Letters, vol. 27, no. 4, 2019.

[24] H. K. Khalil, "Nonlinear Systems," New York, Pearson, 2001.

[25] A. S. Al-Obeidi and S. F. Al-Azzawi, "Chaos synchronization in a 6-D hyperchaotic system with self-excited attractor," Telkomnika Telecommunication, Computing, Electronics and Control, vol. 18, no. 3, pp. 1483-1490, 2020.

[26] Sukono Sambas, A., He, S., Liu, H., Vaidyanathan, S., Hidayat, Y., and Saputra, J., "Dynamical analysis and adaptive fuzzy control for the fractional-order financial risk chaotic system," Advances in Difference Equations, vol. 674, no. 1, pp. 1-12, 2020.

[27] M. E. Yamakou, "Chaotic synchronization of memristive neurons: Lyapunov function versus Hamilton function," Nonlinear Dynamics, vol. 101, no. 1, pp. 487-500, 2020.

\section{BIOGRAPHIES OF AUTHORS}

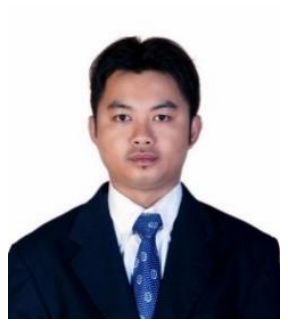

Aceng Sambas is currently a Lecturer at the Muhammadiyah University of Tasikmalaya, Indonesia since 2015. He received his M.Sc in Mathematics from the Universiti Sultan Zainal Abidin (UniSZA), Malaysia in 2015. His current research focuses on dynamical systems, chaotic signals, electrical engineering, computational science, signal processing, robotics, embedded systems and artificial intelligence 

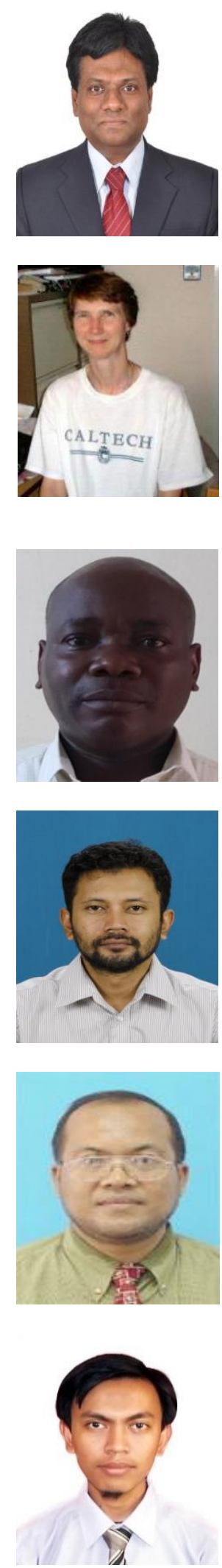

Sundarapandian Vaidyanathan is a Professor at the Research and Development Centre, Vel Tech University, Chennai, India. He earned his D.Sc. in Electrical and Systems Engineering from the Washington University, St. Louis, USA in 1996. His current research focuses on control systems, chaotic and hyperchaotic systems, backstepping control, sliding mode control, intelligent control, computational science and robotics. He has published three text-books on mathematics and twelve research books on control engineering. He has published over 520 Scopus-indexed research publications. He has also conducted many workshops on control systems and chaos theory using MATLAB and SCILAB.

Irene Moroz graduated with a Class I in Mathematics from Oxford University in 1977. She then received her PhD on slowly varying baroclinic waves from Leeds University, UK in 1981. After spending 2 years as a post-doctoral fellow in Leeds, she spent 18 months as a Visiting Assistant Professor at Cornell University, before taking up a New Blood lectureship at the University of East Anglia in 1985. Since 1992, she has been a Fellow in Applied Mathematics at St Hilda's College, Oxford. Her research interests include Dynamical Systems, Mathematical Ecology, Geophysical Fluid Dynamics, Dynamo models and climate modelling.

Babatunde A. Idowu is a PhD holder in Physics and a tutor in the Department of Physics, Lagos State University, Ojo, Lagos Nigeria. His main interest is in chaotic systems, their control and synchronisation. He has published research papers in many national and international journals.

Mohamad Afendee Mohamed received his $\mathrm{PhD}$ in Mathematical Cryptography from Universiti Putra Malaysia in 2011 and currently serves as a senior lecturer at Universiti Sultan Zainal Abidin. His research interests include both theoretical and application issues in the domain of information security, and mobile and wireless networking.

Mustafa Mamat is currently a Professor and the Dean of Graduate School at Universiti Sultan Zainal Abidin (UniSZA), Malaysia since 2013. He was first appointed as a Lecturer at the Universiti Malaysia Terengganu (UMT) in 1999. He obtained his PhD from the UMT in 2007 with specialization in optimization. Later on, he was appointed as a Senior Lecturer in 2008 and then as an Associate Professor in 2010 also at the UMT. To date, he has successfully supervised more than 60 postgraduate students and published more than 150 research papers in various international journals and conferences. His research interests include conjugate gradient methods, steepest descent methods, Broydens family and quasi-Newton methods.

Mada Sanjaya WS received his Ph.D in Mathematics from the University Malaysia Terengganu, Malaysia in 2012. He was first appointed as a Lecturer at the UIN Sunan Gunung Djati Bandung, Indonesia in 2009. His research interests include nonlinear dynamical systems, chaotic systems, artificial intelligence, soft computing and robotic systems. 